\title{
Virtual bilepton effects in polarized Møller scattering
}

\author{
B. Meirose and A. J. Ramalho \\ Instituto de Física \\ Universidade Federal do Rio de Janeiro \\ Caixa Postal 68528, 21945-970 Rio de Janeiro RJ, Brazil
}

October 29, 2018

\begin{abstract}
We investigate the indirect effects of heavy vector bileptons being exchanged in polarized Møller scattering, at the next generation of linear colliders. Considering both longitudinal and transverse beam polarization, and accounting for initial state radiation, beamstrahlung and beam energy spread, we discuss how angular distributions and asymmetries can be used to detect clear signals of virtual bileptons, and the possibility of distinguishing theoretical models that incorporate these exotic particles. We then estimate $95 \%$ C. L. bounds on the mass of these vector bileptons and on their couplings to electrons.
\end{abstract}




\section{Introduction}

Several schemes have been put forward to address important questions left unanswered in the standard model. There are models in which some of the standard model fundamental particles, such as quarks and leptons, are thought to be in fact composite. Another approach is to extend the symmetry by enlarging the local gauge group. In an effort to incorporate gravity, more exotic proposals have been made, such as those which depend on the existence of extra spatial dimensions. Since the standard model has passed severe experimental tests at the $e^{+} e^{-}$and hadron colliders, showing its validity up to the electroweak scale, one expects that it could be seen as an effective theory, valid up to some large mass scale $\Lambda$. Most theoretical arguments point to $\Lambda \gtrsim 1 \mathrm{TeV}$. Extended electroweak models predict the existence of new particles and interactions. An interesting class of exotic particles known as bileptons [1] is present in several of these models, such as left-right symmetric models, technicolor and theories of grand unification. The bileptons in which we are interested are vector bosons which couple to standard model leptons and carry two units of lepton number. In particular, heavy gauge bileptons may be found in models where the standard $S U(2)_{L} \times U(1)$ group is embedded in a larger gauge group. This is the case of some of the 331 gauge models [2]. In this paper we propose to look for indirect signals of doubly-charged vector bileptons in Møller scattering, with polarized beams, and at the new linear collider energies. The next generation of $e^{+} e^{-}$colliders will be designed to operate at center-of-mass energies ranging from the $Z^{0}$ mass to a few $\mathrm{TeV}$, with very high luminosities. Electron and positron beam polarizations are expected to be available at these facilities, providing experimentalists with powerful tools to carry out precision tests of the standard model and to explore new physical phenomena. Currently, the best lower bound on the vector bilepton mass is $M_{Y}>850 \mathrm{GeV}$, a result which was established from muonium-antimuonium conversion [3]. Another useful lower bound $M_{Y}>740 \mathrm{GeV}$ has been derived [4] from current experimental limits on fermion pair production at LEP and lepton-flavor violating charged lepton decays. While less stringent, this limit does not depend on the assumption that the bilepton coupling is flavor-diagonal.

Studies of bilepton effects in Møller scattering have been made before [5], mostly in the context of SU(15) grand unified theories. In this paper we work in the framework of the 331 models, concentrating on the minimal version, and extend the previous studies by taking into account (i) important beam effects such as initial-state radiation, beamstrahlung and beam energy spread; (ii) longitudinal and transverse polarization of the colliding beams, which are expected to be available in the next generation of linear colliders; (iii) Gaussian smearing of the four-momenta of the final-state leptons, simulating the uncertainties in the energy measurements in the electromagnetic calorimeters. All these items are significant for a realistic comparison with experimental data. We analyze several distributions and asymmetries in Møller scattering at the Next Linear Collider (NLC) energies, with the purpose of searching for indirect signals of gauge bileptons. The predictions of an $S U(15)$ GUT model [6] for these observables are also shown for comparison. From the angular distribution of final-state electrons we establish bounds on the couplings and mass of these bileptons at the $95 \%$ confidence level. If evidence of the existence of these vector bileptons is found in Møller scattering, one can also verify the validity of relations between the masses 
of the vector bileptons and new neutral gauge bosons, which are connected to the Higgs structure of the 331 models. In section II we give a brief review of the 331 models [7], outlining only those features that are most relevant to our analysis. Section III describes in detail the numerical simulation of the Møller events and the corresponding analysis of the results. $95 \%$ confidence level limits are established in section IV. Our conclusions are summarized in section $\mathrm{V}$.

\section{Review of the 331 models}

The 331 models are based on the $S U(3)_{C} \otimes S U(3)_{L} \otimes U(1)_{X}$ gauge group and predict new physics at the TeV energy scale. They arrange the ordinary leptons in $S U(3)_{L}$ antitriplets, two generations of quarks in triplets and a third generation in an antitriplet. Anomaly cancelation, essential to a gauge theory, takes place not within each generation but when all three families of quarks and leptons are taken together. The fact that one quark generation transforms differently from the other two is essential in these models, since the number of triplets must equal the number of antitriplets to ensure that the models remain anomaly-free. This implies that the number of generations is divisible by the number of colors. Besides standard gauge bosons, the models predict a new neutral gauge boson $Z^{\prime}$ and four vector bileptons $Y^{ \pm}$and $Y^{ \pm \pm}$. These gauge bosons are expected to be considerably heavier than the standard gauge bosons. In addition to the ordinary quarks, the class of models we are considering here contains three new heavy quarks, with exotic electric charges $5 / 3$ and $-4 / 3$. However it is possible to build 331 models in which these exotic quarks are not present [8], and as a consequence, neither are doubly-charged gauge bileptons. Following the notation of ref. [5], the coupling of the doubly-charged vector bileptons $Y^{++}$to electrons and positrons is given by the interaction Lagrangian

$$
L_{i n t}=-\frac{g_{3 l}}{\sqrt{2}} Y_{\mu}^{++} e^{T} C \gamma^{\mu} \gamma_{5} e-\frac{g_{3 l}}{\sqrt{2}} Y_{\mu}^{--} \bar{e} \gamma^{\mu} \gamma_{5} C \bar{e}^{T}
$$

For the minimal 331 model, symmetry breaking can be accomplished by three complex $S U(3)_{L}$ triplets and a complex sextet, allowing these nonstandard gauge bosons to acquire plausible masses. In this case, the mass $M_{Z^{\prime}}$ of the neutral vector boson $Z^{\prime}$ and the mass $M_{Y}$ of the doubly-charged bilepton $Y^{++}$are related by

$$
\frac{M_{Y}}{M_{Z^{\prime}}}=\frac{\sqrt{3\left(1-4 \sin ^{2} \theta_{W}\right)}}{2 \cos \theta_{W}}
$$

Alternative Higgs structures are possible in different 331 models, but then the relation above no longer holds.

\section{Simulation and analysis}

One substantial source of beam energy degradation is the initial-state radiation (ISR). This is a QED effect, and corresponds to the emission of photons by the incoming electrons and 
positrons. For a considerable fraction of events, this bremsstrahlung emission lowers the effective center-of-mass energy available for the hard scattering process. To account for ISR, we used the structure function approach discussed in [9]. In order to achieve high luminosities at the new linear colliders, the beams must have very small transverse dimensions. The particles in a colliding bunch suffer considerable transverse acceleration due to the collective electromagnetic fields produced by the particles in the opposite colliding bunches, which gives rise to the emission of synchrotron radiation, the so-called beamstrahlung. The effective energy available for the reaction is then smaller than the nominal value. The average energy loss of a positron or electron by beamstrahlung depends on the design parameters of the accelerator. For some designs of a $1 \mathrm{TeV} \mathrm{NLC}$, for instance, the colliding beams may lose about $13 \%$ of the nominal energy from beamstrahlung emissions. This loss may reach $31 \%$ at a $3 \mathrm{TeV}$ CLIC [10]. To obtain the beamstrahlung spectrum, we followed the approach of ref. [11, which is based on the Yokoya-Chen approximate evolution equation for beamstrahlung [12]. In this paper, the calculations of the beamstrahlung energy spectra were carried out starting from the energy-dependent sets of NLC design parameters [13]. The spectra corresponding to ISR and beamstrahlung emissions were convoluted, and the resulting distribution was used to compute all the required differential cross sections. We also considered a possible beam energy spread, which was taken to be Gaussian distributed, with a width of $1 \%$ of the nominal beam energy.

In addition to the standard Feynman diagrams for Møller scattering, in 331 models the process also proceeds via an s-channel exchange of a doubly-charged bilepton, and a t-channel exchange of a $Z^{\prime}$ boson as well. By ignoring beam effects, neglecting the $Z^{\prime}$ exchanges and considering only unpolarized beams, we verified that our numerical calculations agree with the trace calculation of ref. [5]. Likewise, we cross-checked our calculations with those of ref. [14], which were carried out in the framework of the standard model, but with arbitrary beam polarization.

The differential cross sections were calculated with Monte Carlo techniques, with the simulated events selected according to the following set of cuts : (i) the final-state electrons and positrons were required to be produced within the angular range $\left|\cos \theta_{i}\right|<0.95$, where $\theta_{i}$ stands for the polar angle of the final-state lepton with respect to the direction of the incoming electron beam; (ii) all events in which the acollinearity angle $\zeta$ of the final-state $e^{+}-e^{-}$three-momenta did not pass the cut $\zeta<10^{\circ}$ were rejected; (iii) the ratio of the effective center-of-mass energy to the nominal center-of-mass energy for any acceptable event was required to be greater than 0.9 . Our simulations were made in the context of the NLC program [15], considering a maximum center-of-mass energy of $1 \mathrm{TeV}$, and several years of operation, so as to accumulate an integrated luminosity of $500 \mathrm{fb}^{-1}$. In order to simulate the finite resolution of the NLC electromagnetic calorimeters, we Gaussian-smeared the fourmomenta of the produced electrons and positrons [16]. The energies of the final-state leptons were distributed as a Gaussian with half-width $\Delta E$ of the form $\Delta E / E=10 \% / \sqrt{E} \oplus 1 \%$, whereas the directions of the lepton three-momenta were smeared in a cone around the corresponding original directions, whose half-angle is a Gaussian with half-width equal to 10 mrad.

Beam polarization will play a useful role at the new linear colliders [17]. By using polarized lepton beams, one can effectively reduce backgrounds and increase the sensitivity of spin- 
dependent observables to potential new physics. In our calculations we worked with the electron beam projection operators in the extreme relativistic regime,

$$
\begin{gathered}
\mathcal{P}\left(p_{a, b}\right)=\lim _{m \rightarrow 0} \frac{1}{2}\left(\not p_{a, b}+m\right)\left(1+\gamma_{5} \not h_{a, b}\right) \rightarrow \\
\frac{1}{2}\left(1+P_{L}^{a, b} \gamma_{5}\right) \not p_{a, b}+\frac{1}{2} P_{T}^{a, b} \gamma_{5}\left(\cos \phi_{a, b} \not h_{1}+\sin \phi_{a, b} \not h_{2}\right) \not \not_{a, b},
\end{gathered}
$$

where $n_{a, b}^{\mu}$ represent the spin vectors and $P_{L}^{a, b}\left(P_{T}^{a, b}\right)$ stand for the longitudinal (transverse) polarizations of the incoming electron beams, whose four-momenta for a nominal center-ofmass energy $\sqrt{s}$ are given by $p_{a}^{\mu}=\left(\frac{\sqrt{s}}{2}, 0,0, \frac{\sqrt{s}}{2}\right)$ and $p_{b}^{\mu}=\left(\frac{\sqrt{s}}{2}, 0,0,-\frac{\sqrt{s}}{2}\right)$. For the numerical calculations dealing with transverse polarization of the electron beams, the azimuthal angles of the transverse polarization vectors were taken to be $\phi_{a}=\phi_{b}=0$, and the purely spatial vectors $n_{1}^{\mu}=(0,1,0,0)$ and $n_{2}^{\mu}=(0,0,1,0)$.

In order to discuss how to probe the presence of gauge bileptons in Møller scattering, we first considered longitudinally polarized electron beams. For the longitudinal polarizations of the beams we considered $P_{L}^{a}=-P_{L}^{b}=0.9$, with an uncertainty given by $\Delta P_{L} / P_{L}=0.5 \%$. Figure 1 shows the dependence of the total cross section on the center-of-mass energy, for an input bilepton mass $M_{Y}=1.2 \mathrm{TeV}$. Only for higher energy values is the total cross section significantly altered by the exchange of a vector bilepton. The angular distribution $d \sigma / d(\cos \theta)$ of the final-state electrons is, however, more sensitive to the presence of such a particle. This is displayed in Fig. 2, where the angular distribution is plotted both for $\sqrt{s}=500 \mathrm{GeV}$ and $\sqrt{s}=1 \mathrm{TeV}$, with the corresponding curves for the standard model shown for comparison. The Møller scattering angular distribution in the minimal 331 model differs from that of the standard model for most of the angular range, the more so for a center-of-mass energy $\sqrt{s}=1 \mathrm{TeV}$. These deviations from the standard model predictions can be used to establish bounds on the mass of the gauge bilepton, and its coupling to electrons. The symmetric shape of the angular distribution suggests that the integrated forward-backward asymmetry $A_{F B}$ should be small. This is indeed the case, as shown in Fig. 3, where $A_{F B}$ is plotted for several input values of $M_{Y}$, at an energy $\sqrt{s}=1 T e V$, and the one-standard-deviation error bars represent only the statistical errors. Next we analyze the discovery potential of spin asymmetries.

Starting from the polarization-dependent angular distributions, one can compute the following asymmetries:

$$
\begin{gathered}
A_{1}(\cos \theta)=\frac{d \sigma\left(-\left|P_{L}^{a}\right|,-\left|P_{L}^{b}\right|\right)+d \sigma\left(-\left|P_{L}^{a}\right|,\left|P_{L}^{b}\right|\right)-d \sigma\left(\left|P_{L}^{a}\right|,-\left|P_{L}^{b}\right|\right)-d \sigma\left(\left|P_{L}^{a}\right|,\left|P_{L}^{b}\right|\right)}{d \sigma\left(-\left|P_{L}^{a}\right|,-\left|P_{L}^{b}\right|\right)+d \sigma\left(-\left|P_{L}^{a}\right|,\left|P_{L}^{b}\right|\right)+d \sigma\left(\left|P_{L}^{a}\right|,-\left|P_{L}^{b}\right|\right)+d \sigma\left(\left|P_{L}^{a}\right|,\left|P_{L}^{b}\right|\right)} \\
A_{2}(\cos \theta)=\frac{d \sigma\left(-\left|P_{L}^{a}\right|,-\left|P_{L}^{b}\right|\right)-d \sigma\left(\left|P_{L}^{a}\right|,\left|P_{L}^{b}\right|\right)}{d \sigma\left(-\left|P_{L}^{a}\right|,-\left|P_{L}^{b}\right|\right)+d \sigma\left(\left|P_{L}^{a}\right|,\left|P_{L}^{b}\right|\right)} \\
A_{3}(\cos \theta)=\frac{d \sigma\left(-\left|P_{L}^{a}\right|,\left|P_{L}^{b}\right|\right)-d \sigma(0,0)}{d \sigma\left(-\left|P_{L}^{a}\right|,\left|P_{L}^{b}\right|\right)+d \sigma(0,0)}
\end{gathered}
$$

In the limit $\left|P_{L}^{a}\right|=\left|P_{L}^{b}\right|=1, A_{1}(\cos \theta)$ and $A_{2}(\cos \theta)$ reduce to the familiar parity violating Møller asymmetries [18]

$$
A_{L R}^{(1)}=\frac{d \sigma_{L L}+d \sigma_{L R}-d \sigma_{R L}-d \sigma_{R R}}{d \sigma_{L L}+d \sigma_{L R}+d \sigma_{R L}+d \sigma_{R R}}
$$


and

$$
A_{L R}^{(2)}=\frac{d \sigma_{L L}-d \sigma_{R R}}{d \sigma_{L L}+d \sigma_{R R}}
$$

respectively.

The behavior of spin asymmetry $A_{1}$ as a function of $\cos \theta$ is depicted in Fig. 4, for a bilepton mass $M_{Y}=1.2 \mathrm{TeV}$. At $\sqrt{s}=500 \mathrm{GeV}$ the deviation from the standard model values is small. This asymmetry becomes more sensitive to the presence of a vector bilepton at an energy of $1 \mathrm{TeV}$, and its angular dependence distinguishes the two nonstandard models . Statistical errors for the spin asymmetries discussed in this section are rather small, and most of the effects of the systematic errors are expected to cancel out in these asymmetries. Fig. 5 shows that a similar pattern holds for $A_{2}$, which is represented as a function of $\cos \theta$ for both $500 \mathrm{GeV}$ and $1 \mathrm{TeV}$. At this latter energy, however, $A_{2}$ does not lead to a clear distinction between the minimal 331 model and the $S U(15)$ GUT model. Asymmetry $A_{3}$ concerns the difference between a polarized angular distribution and its unpolarized counterpart, and is displayed in Fig. 6. Unlike $A_{1}$ and $A_{2}$, which take values of the order of $5 \%, A_{3}$ may become quite large, reaching a maximum magnitude of about $63 \%$ for the minimal 331 model at $\sqrt{s}=1 \mathrm{TeV}$. It can be useful to discriminate a model with vector bileptons from the standard model, even at a $500 \mathrm{GeV}$ linear collider. At a center-of-mass energy of $1 \mathrm{TeV}$, the difference between the prediction of the 331 minimal model for $A_{3}$ and the standard values is even more striking.

We assume that in the next generation of linear colliders, transverse polarization of electron beams will be available as an extra tool to search for the new physics. This could be achieved by means of spin rotators, which convert longitudinal into transverse polarization. It is not immediately clear whether the use of transversely polarized electron beams may add any important information, which could not be extracted from longitudinally polarized Møller scattering. In fact, standard Møller scattering is known not to be particularly sensitive to transverse beam polarization [14, but the presence of an s-channel vector bilepton might modify this picture. Effects of transverse beam polarization in the nonstandard Møller scattering under discussion only materialize if both beams are transversely polarized. In order to search for possible advantages of transverse beam polarization for the problem at hand, we examined in detail the behavior of the following differential azimuthal asymmetry:

$$
A_{1 T}(\cos \theta)=\frac{\int_{(+)} d \phi \frac{d^{2} \sigma}{d(\cos \theta) d \phi}-\int_{(-)} d \phi \frac{d^{2} \sigma}{d(\cos \theta) d \phi}}{\int_{(+)} d \phi \frac{d^{2} \sigma}{d(\cos \theta) d \phi}+\int_{(-)} d \phi \frac{d^{2} \sigma}{d(\cos \theta) d \phi}},
$$

where the subscript $+(-)$ indicates that the integration over the azimuthal angle $\phi$ is to be carried out over the region of phase space where $\cos 2 \phi$ is positive (negative). As far as $A_{1 T}(\cos \theta)$ is concerned, and considering an integrated luminosity of $500 \mathrm{fb}^{-1}$, we found that it would be difficult to separate the signal from the standard model background at centerof-mass energies around $500 \mathrm{GeV}$, even if $M_{Y}$ is only moderately large. At $\sqrt{s}=1 \mathrm{TeV}$, however, it is feasible to detect the effects of a vector bilepton, as long as its mass is not much larger than the center-of-mass energy. This is illustrated in Fig. 7, where $A_{1 T}(\cos \theta)$ is plotted both for an input mass $M_{Y}=800 \mathrm{GeV}$ and and for $M_{Y}=1.2 \mathrm{TeV}$, along with the standard model expectation. We also investigated the integrated version of the asymmetry 
above,

$$
A_{2 T}=\frac{\int_{(+)} d(\cos \theta) d \phi \frac{d^{2} \sigma}{d(\cos \theta) d \phi}-\int_{(-)} d(\cos \theta) d \phi \frac{d^{2} \sigma}{d(\cos \theta) d \phi}}{\int_{(+)} d(\cos \theta) d \phi \frac{d^{2} \sigma}{d(\cos \theta) d \phi}+\int_{(-)} d(\cos \theta) d \phi \frac{d^{2} \sigma}{d(\cos \theta) d \phi}}
$$

where the integrations are consistent with the cuts specified in section III. The mass dependence of $A_{2 T}$ for $\sqrt{s}=500 \mathrm{GeV}$ and $\sqrt{s}=1 \mathrm{TeV}$ is presented in Fig. 8. $A_{2 T}$ is found to be sizable over a fairly wide range around the bilepton resonance. We checked that the shape of the curve representing the mass dependence of this asymmetry becomes wider as $g_{3 l}$ increases, while the position of the corresponding minimum remains essentially the same, for a fixed center-of-mass energy.

\section{Bounds}

A $\chi^{2}$ test was applied to estimate discovery limits for a vector bilepton in Møller scattering. Considering only longitudinal beam polarization, we compared the angular distribution $d \sigma / d(\cos \theta)$ of the final-state electrons, modified by the presence of a vector bilepton, with the corresponding standard model distribution. Assuming that the experimental data will be well described by the standard model predictions, we defined a two-parameter $\chi^{2}$ estimator

$$
\chi^{2}\left(g_{3 l}, M_{Y}\right)=\sum_{i=1}^{N_{b}}\left(\frac{N_{i}^{S M}-N_{i}}{\Delta N_{i}^{S M}}\right)^{2}
$$

where $N_{i}^{S M}$ is the number of standard model events detected in the $i^{\text {th }}$ bin, $N_{i}$ is the number of events in the $i^{\text {th }}$ bin as predicted by the model with bileptons, and $\Delta N_{i}^{S M}=$ $\sqrt{\left(\sqrt{N_{i}^{S M}}\right)^{2}+\left(N_{i}^{S M} \epsilon\right)^{2}}$ the corresponding total error, which combines in quadrature the Poisson-distributed statistical error with the systematic error. For the latter we assumed a conservative value $\epsilon=5 \%$ for each measurement. The angular range $|\cos \theta|<0.95$ was divided into $N_{b}=20$ equal-width bins. The coupling $g_{3 l}$ of a vector bilepton to electrons and the bilepton mass $M_{Y}$ were varied as free parameters to determine the $\chi^{2}$ distribution. The $95 \%$ confidence level bound corresponds to an increase of the $\chi^{2}$ by 5.99 with respect to the minimum $\chi_{\min }^{2}$ of the distibution. Fig.9 presents the resulting $95 \%$ C. L. contour plots on the $\left(g_{3 l}, M_{Y}\right)$ plane for the nominal center-of-mass energies $\sqrt{s}=500 \mathrm{GeV}$ and $\sqrt{s}=1 \mathrm{TeV}$. The unpolarized case is shown in Fig. 10. Since our differential cross sections contain only even powers of $g_{3 l}$, it suffices to use positive values of the coupling in Figs. 9-10. We also calculated the corresponding $95 \%$ C. L. limits on the bilepton mass at these NLC energies, considering only the minimal 331 model, in which a $Z^{\prime}$ exchange has to be taken into account. The results are displayed in Table I.

\section{Conclusions}

Future linear colliders will provide an opportunity to look for new particles and their interactions. In this paper we discussed how to search for effects of a virtual vector bilepton 
Table 1: $95 \%$ C. L. limits on the bilepton mass in the minimal 331 model, at NLC energies

\begin{tabular}{lcc} 
Polarization & $\sqrt{s}=500 \mathrm{GeV}$ & $\sqrt{s}=1 \mathrm{TeV}$ \\
\hline unpolarized & $1230 \mathrm{GeV}$ & $1815 \mathrm{GeV}$ \\
polarized & $2529 \mathrm{GeV}$ & $4574 \mathrm{GeV}$
\end{tabular}

in polarized Møller scattering. Starting from a realistic simulation of this process, we analyzed several polarization-dependent observables that might provide strong evidence of the existence of vector bileptons, and allow to discriminate models or classes of models which predict these particles, should any deviation from the standard expectations be detected. We demonstrated that these polarization-dependent observables are more sensitive to vector bileptons than their unpolarized counterparts, in a large region of the allowed parameter space. This sensitivity was found to be stronger at a center-of-mass energy of $1 \mathrm{TeV}$ than at $500 \mathrm{GeV}$. The bounds on the masses and couplings of the bileptons, derived from a $\chi^{2}$ estimator, indicate that it should be possible to probe mass scales of up to several $\mathrm{TeV}$ for a signal of a vector bilepton. Longitudinal polarization of the electron beams in Møller scattering has proved useful to improve these bounds on the masses and couplings. Although most of our calculations were carried out in the context of the minimal 331 model and an $S U(15)$ GUT model, we believe that our overall conclusions could be extended to other models with bileptons.

\section{References}

[1] F. Cuypers and S. Davidson, Eur. Phy. J. C 2,503 (1998) hep-ph/9609487].

[2] F. Pisano and V. Pleitez, Phys. Rev. D 46, 410 (1992); P. H. Frampton, Phys. Rev. Lett. 69, 2889 (1992).

[3] L. Willmann et. al., Phys. Rev. Lett. 82, 49 (1999).

[4] M. B. Tully and G. C. Joshi, Phys. Lett. B466, 333 (1993); hep-ph/9905552.

[5] Paul H. Frampton and Daniel Ng, Phys. Rev. D 45, 4240 (1992); Thomas Rizzo, Phys. Rev. D 46, 910 (1992).

[6] P. H. Frampton and B.-H. Lee, Phys. Rev. Lett. 64, 619 (1990); P. H. Frampton, Int. J. Mod. Phys. A15, 2455 (2000).

[7] F. Pisano, V. Pleitez and M. D. Tonasse, IFT-UNESP preprint IFT-P.043/97 and references therein.

[8] W. A. Ponce, J. B. Flores and L. A. Sanchez, Int. J. Mod. Phys. A17, 643 (2002).

[9] M. Skrzypek and S. Jadach, Z. Phys. C49, 577 (1991).

[10] The CLIC Study Team, "A $3 \mathrm{TeV} e^{+} e^{-}$Linear Collider Based on CLIC Technology", report CERN 2000-008 (2000). 
[11] M. Peskin, SLAC-TN-04-032 (1999).

[12] K. Yokoya and P. Chen, Proceedings of the 1989 IEEE Particle Accelerator Conference, edited by F. Bennett and L. Taylor, SLAC-PUB-4935.

[13] Beam Parameters and Lattices for NLC2003, http://www-project.slac.stanford.edu/lc/local/documentation/pdf/NLC2003_config.pdf.

[14] Haakon A. Olsen and Per Osland, Phys. Rev. D25, 2895 (1982).

[15] T. Abe et al., American Linear Collider Working Group, in Proceedings of the APS/DPF/DPB Summer Study on the Future of Particle Physics (Snowmass 2001), ed. N. Graf, hep-ex/0106055.

[16] R. Settles, H. Spiesberger and W. Wiedenmann, Smear version 3.02; http://www.desy.de/hspiesb/smear.html.

[17] G. Moortgat-Pick and H. Steiner, Eur. Phys. J. direct C3 (2001) 1 [hep-ph/0106155].

[18] Andrzej Czarnecki and William Marciano, Int. J. Mod. Phys. A13 (1998) 2235 hep-ph/9801394. 


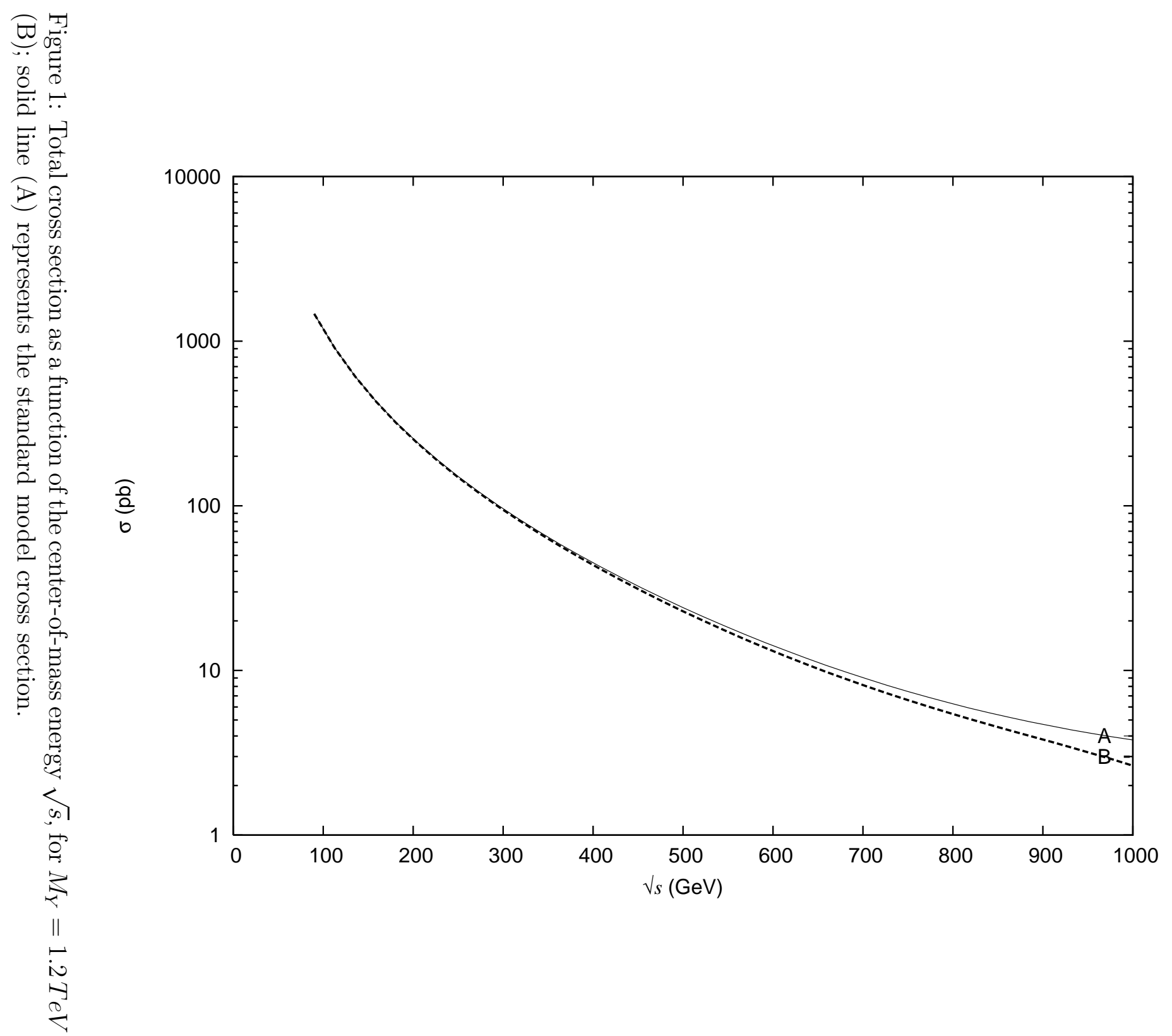




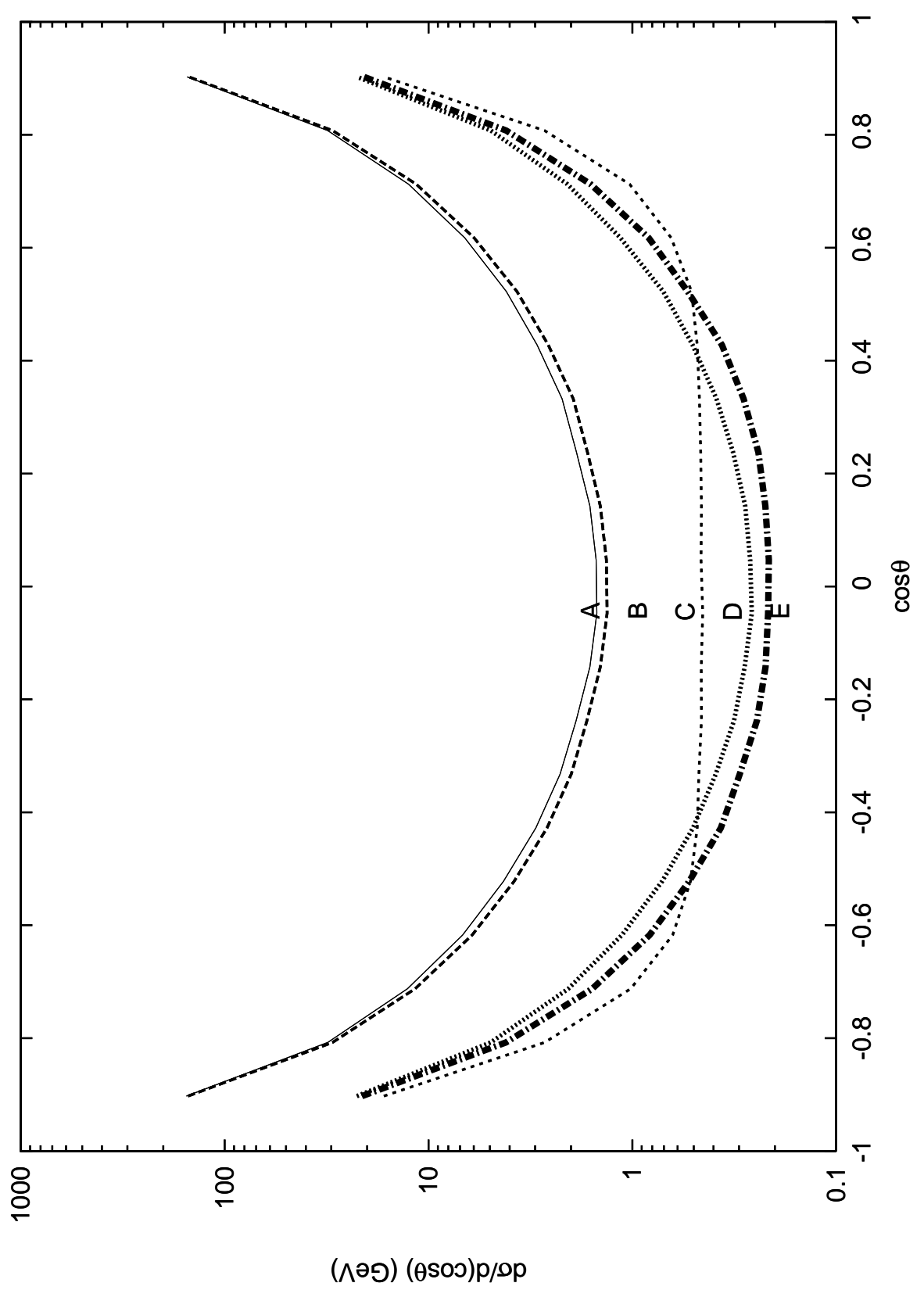

Figure 2: Angular distribution of the final-state electrons; Curves (A) and (D) show the angular spectra predicted by the standard model at $\sqrt{s}=500 \mathrm{GeV}$ and $\sqrt{s}=1 \mathrm{TeV}$ respectively, while (B) and (C) display the corresponding angular distributions in the minimal 331 model. Curve (E) shows the prediction for the $\mathrm{SU}(15)$ model at $\sqrt{s}=1 \mathrm{TeV}$. 


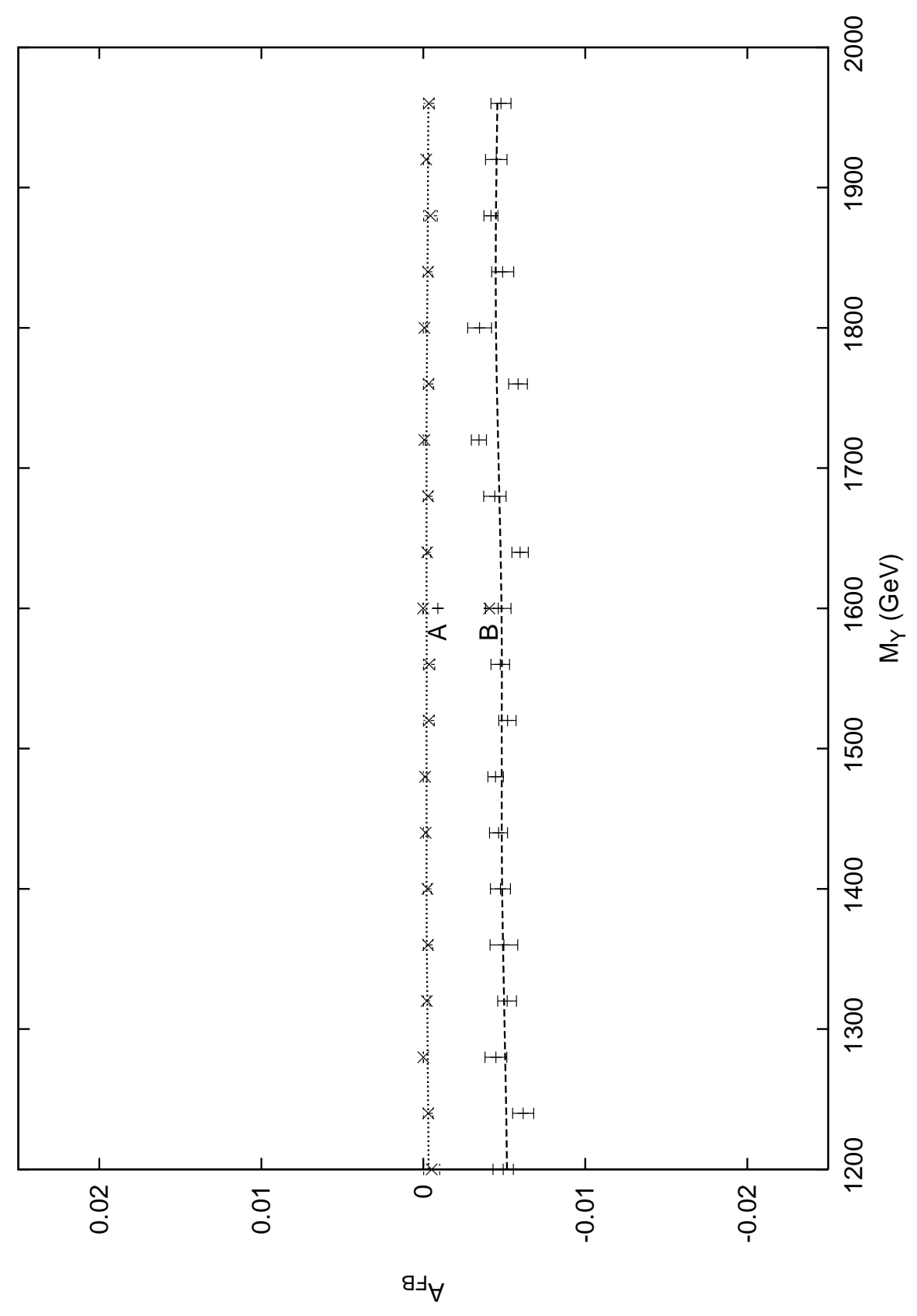

Figure 3: Forward-backward asymmetry $A_{F B}$ for several input masses $M_{Y}$, at $\sqrt{s}=1 T e V$, according to the minimal 331 model (B). The upper curve (A) shows the expected value for the standard model 


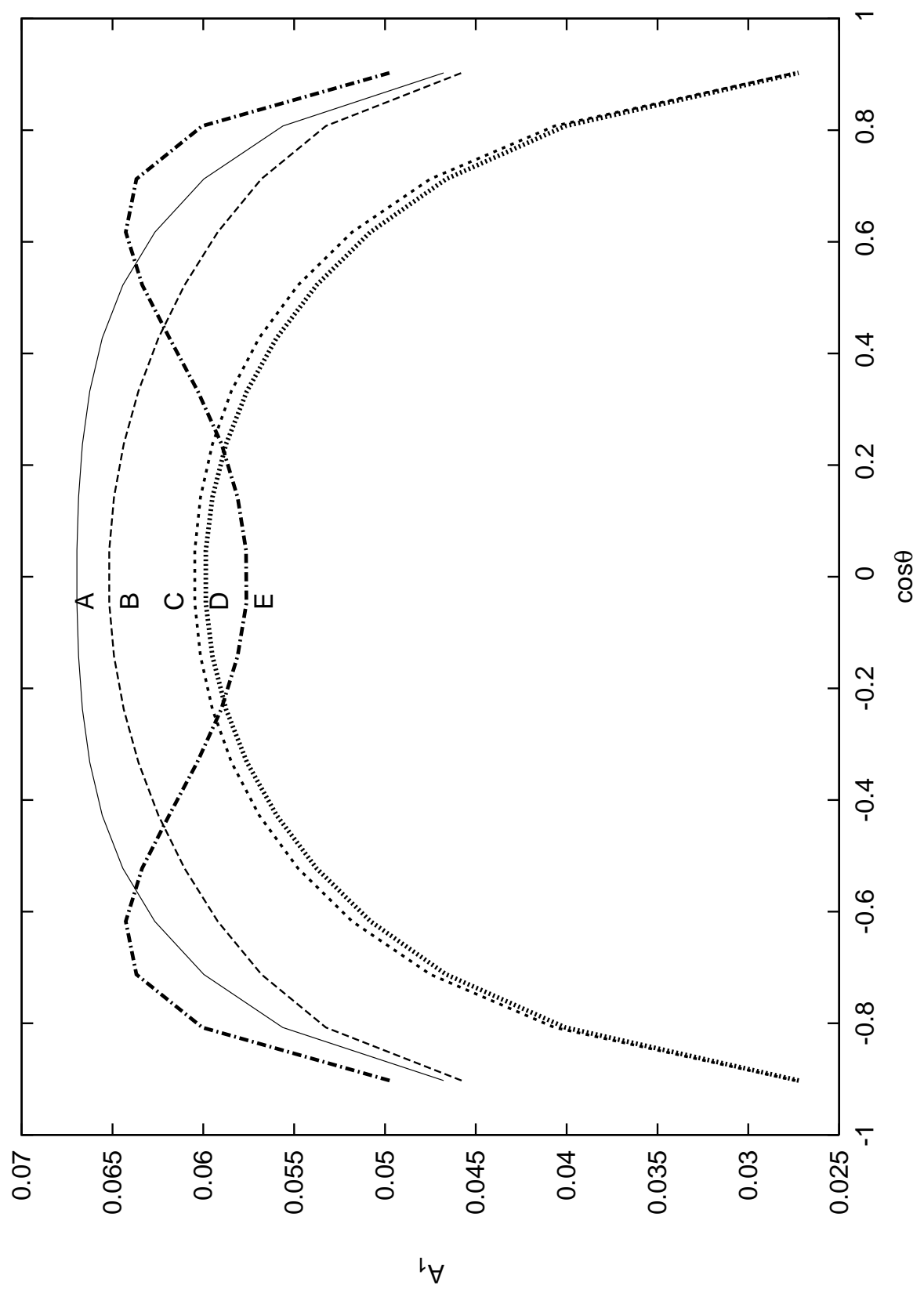

Figure 4: Polar angle dependence of spin asymmetry $A_{1}(\cos \theta)$; Curve $(\mathrm{A})$ shows the prediction for the $\mathrm{SU}(15)$ model at $\sqrt{s}=1 \mathrm{TeV}$. Curves (D) and (B) show the standard model predictions for $A_{1}(\cos \theta)$ at $\sqrt{s}=500 \mathrm{GeV}$ and $\sqrt{s}=1 \mathrm{TeV}$ respectively, while (C) and (E) represent the corresponding expectations for the minimal 331 model, for a mass $M_{Y}=1.2 \mathrm{TeV}$. 


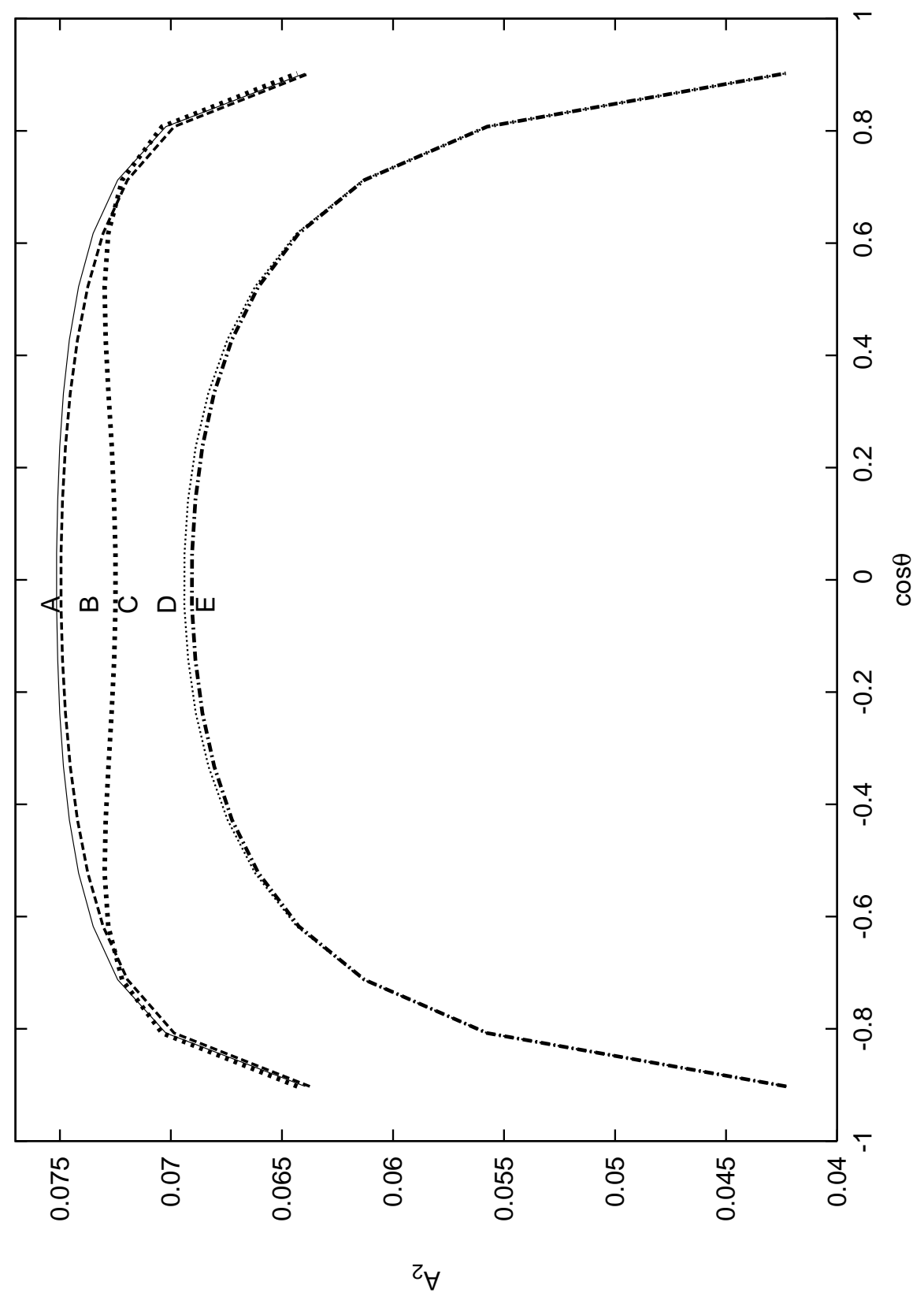

Figure 5: Polar angle dependence of spin asymmetry $A_{2}(\cos \theta)$; Curve $(\mathrm{A})$ shows the prediction for the $\mathrm{SU}(15)$ model at $\sqrt{s}=1 \mathrm{TeV}$. Curves (B) and (D) show the standard model predictions for $A_{2}(\cos \theta)$ at $\sqrt{s}=500 \mathrm{GeV}$ and $\sqrt{s}=1 \mathrm{TeV}$ respectively, while $(\mathrm{C})$ and (E) represent the corresponding expectations for the minimal 331 model, for a mass $M_{Y}=1.2 \mathrm{TeV}$. 


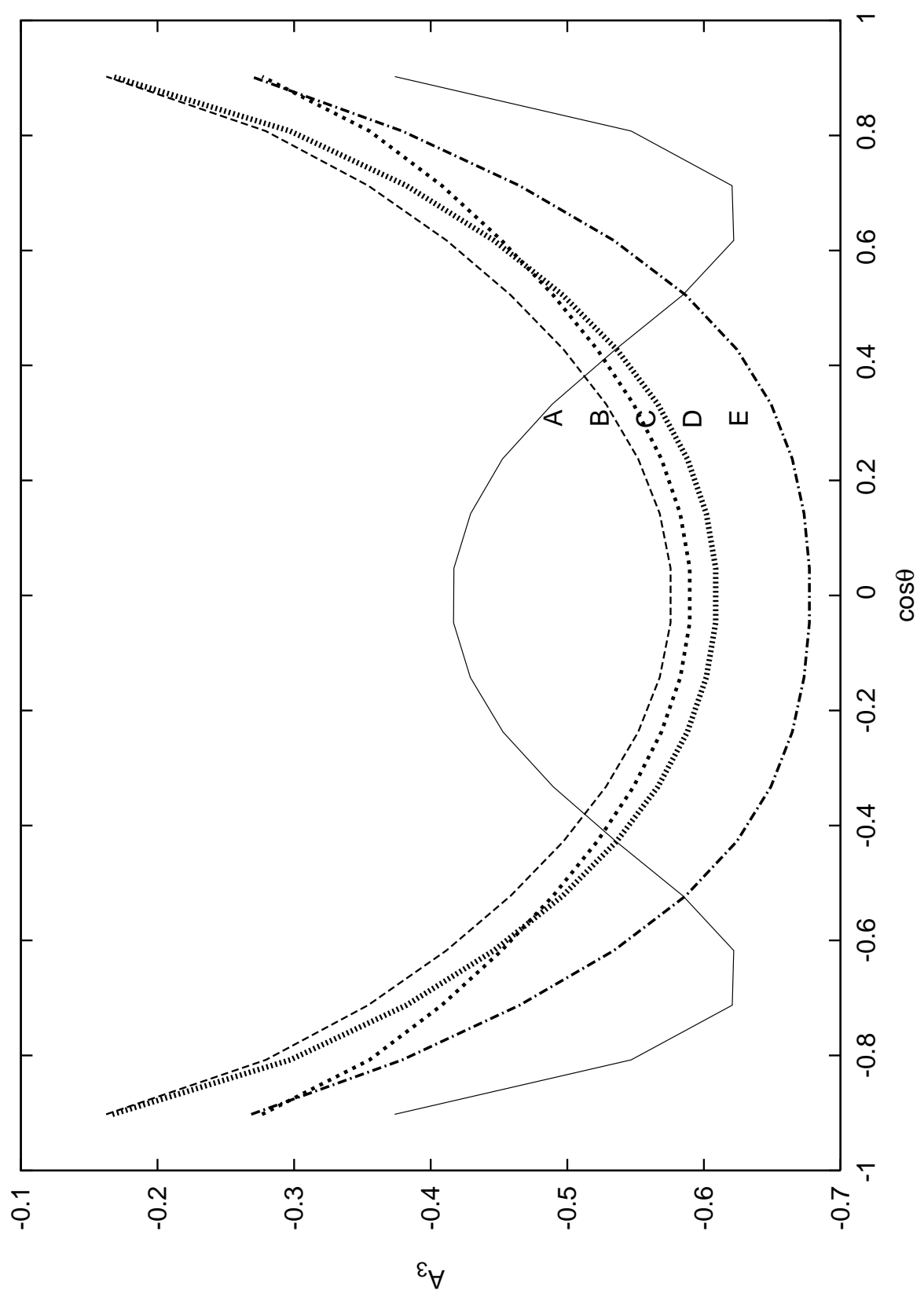

Figure 6: Polar angle dependence of spin asymmetry $A_{3}(\cos \theta)$; Curves $(\mathrm{B})$ and $(\mathrm{C})$ show the standard model predictions for $A_{3}(\cos \theta)$ at $\sqrt{s}=500 \mathrm{GeV}$ and $\sqrt{s}=1 \mathrm{TeV}$ respectively, while (D) and (A) represent the corresponding expectations for the minimal 331 model, for a mass $M_{Y}=1.2 \mathrm{TeV}$. Curve (E) shows the prediction for the $\mathrm{SU}(15)$ model at $\sqrt{s}=1 \mathrm{TeV}$. 


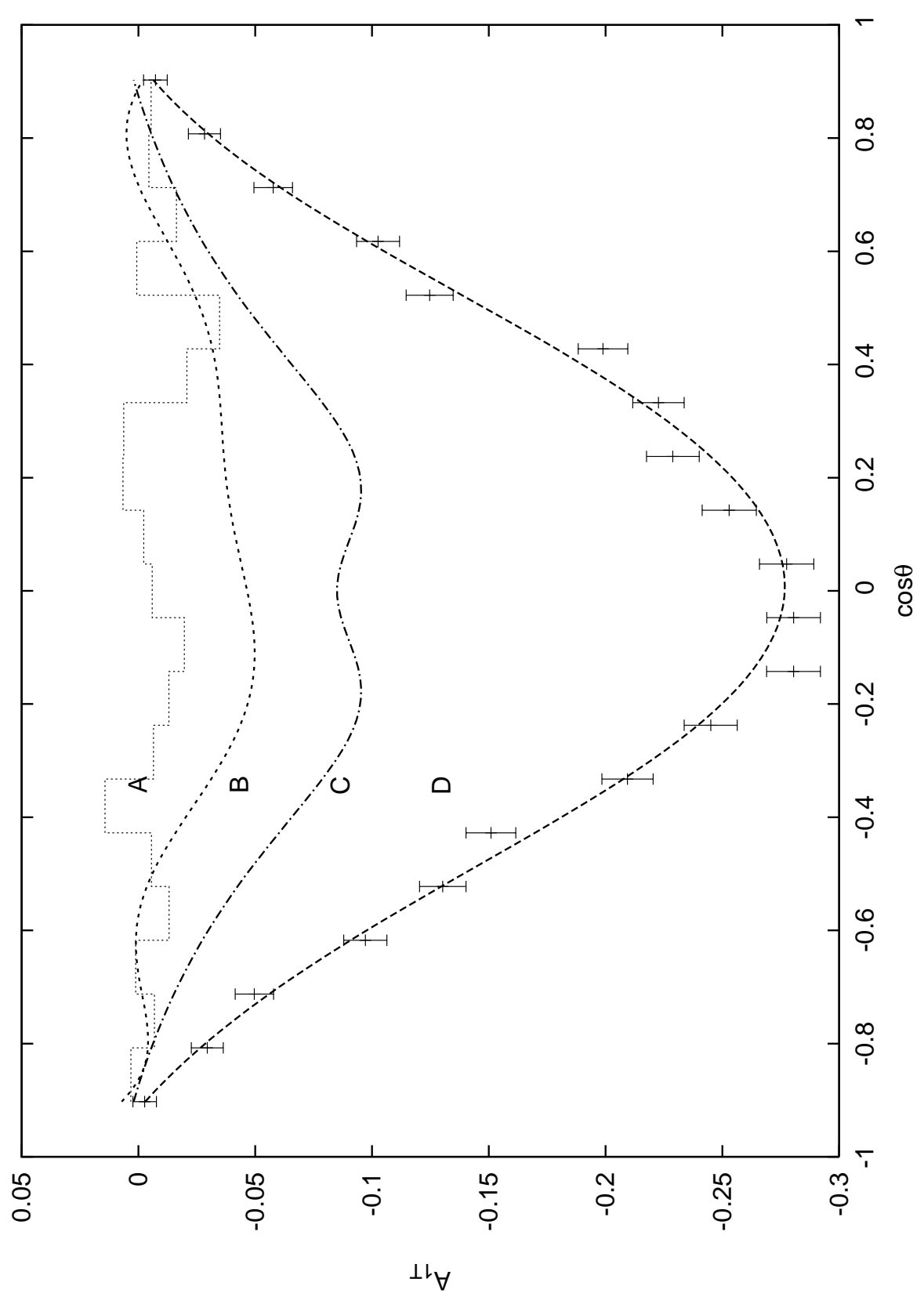

Figure 7: Transverse polarization asymmetry $A_{1 T}(\cos \theta)$ as a function of $\cos \theta$, at $\sqrt{s}=1 T e V$. The curve with error bars (D) corresponds to the minimal 331 model with $M_{Y}=800 \mathrm{GeV}$, whereas curve $(\mathrm{C})$ corresponds to the $S U(15)$ GUT model prediction for the same mass. Curve (B) represents the behavior of the asymmetry for a $1.2 \mathrm{TeV}$ bilepton in the minimal 331 model, and the histogram (A) corresponds to the standard model expectation. The error bars for the upper curves are similar to those of the lower curve on a bin-to-bin basis. 


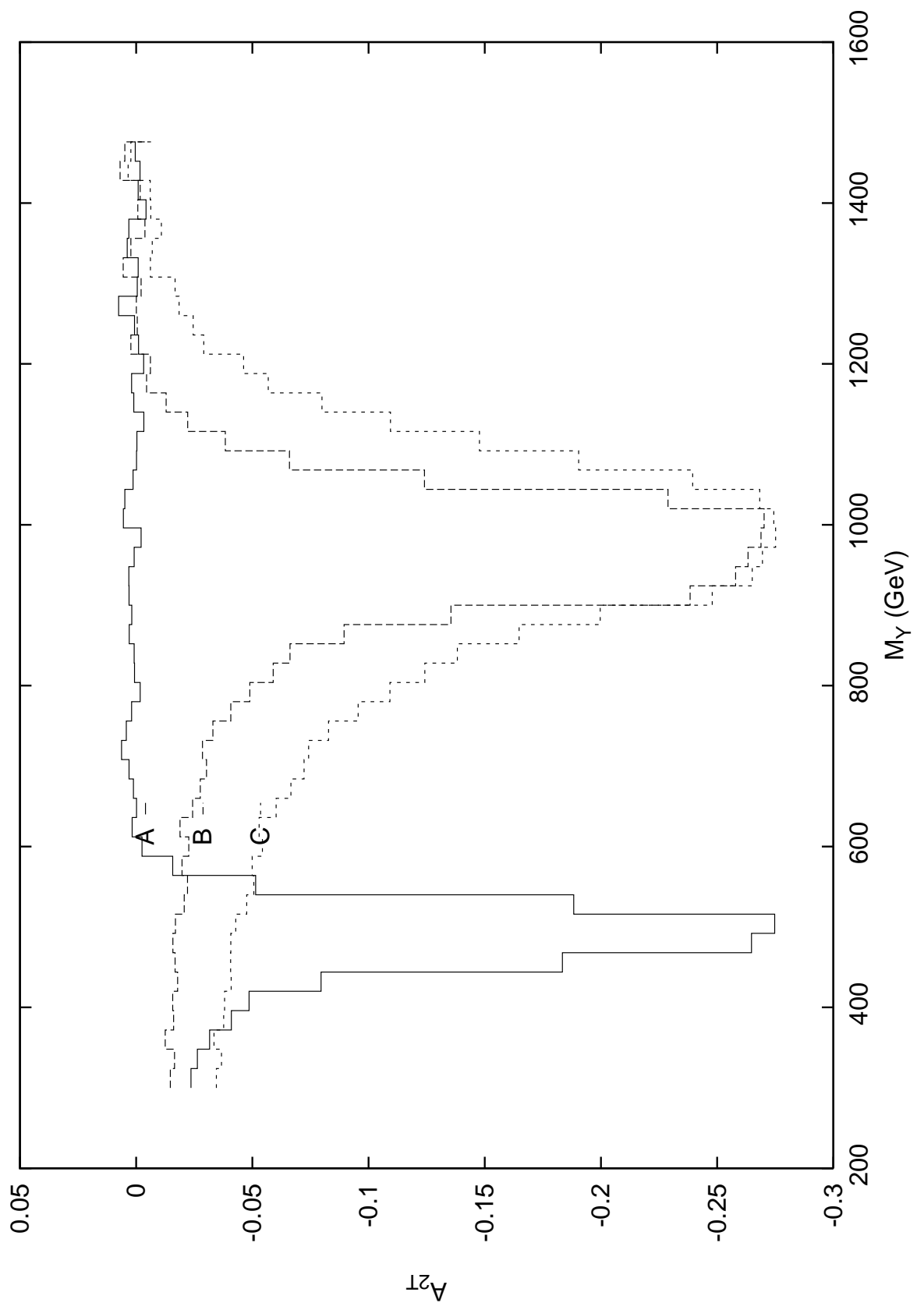

Figure 8: Transverse polarization asymmetry $A_{2 T}$ as a function of $M_{Y}$. The solid histogram (A) refers to the minimal 331 model prediction at an energy $\sqrt{s}=500 \mathrm{GeV}$ and the longdashed histogram (B) to the $S U(15)$ model at $1 \mathrm{TeV}$. The resulting asymmetry $A_{2 T}$ for the minimal 331 model at $\sqrt{s}=1 \mathrm{TeV}$ is is represented by the dashed histogram (C). 


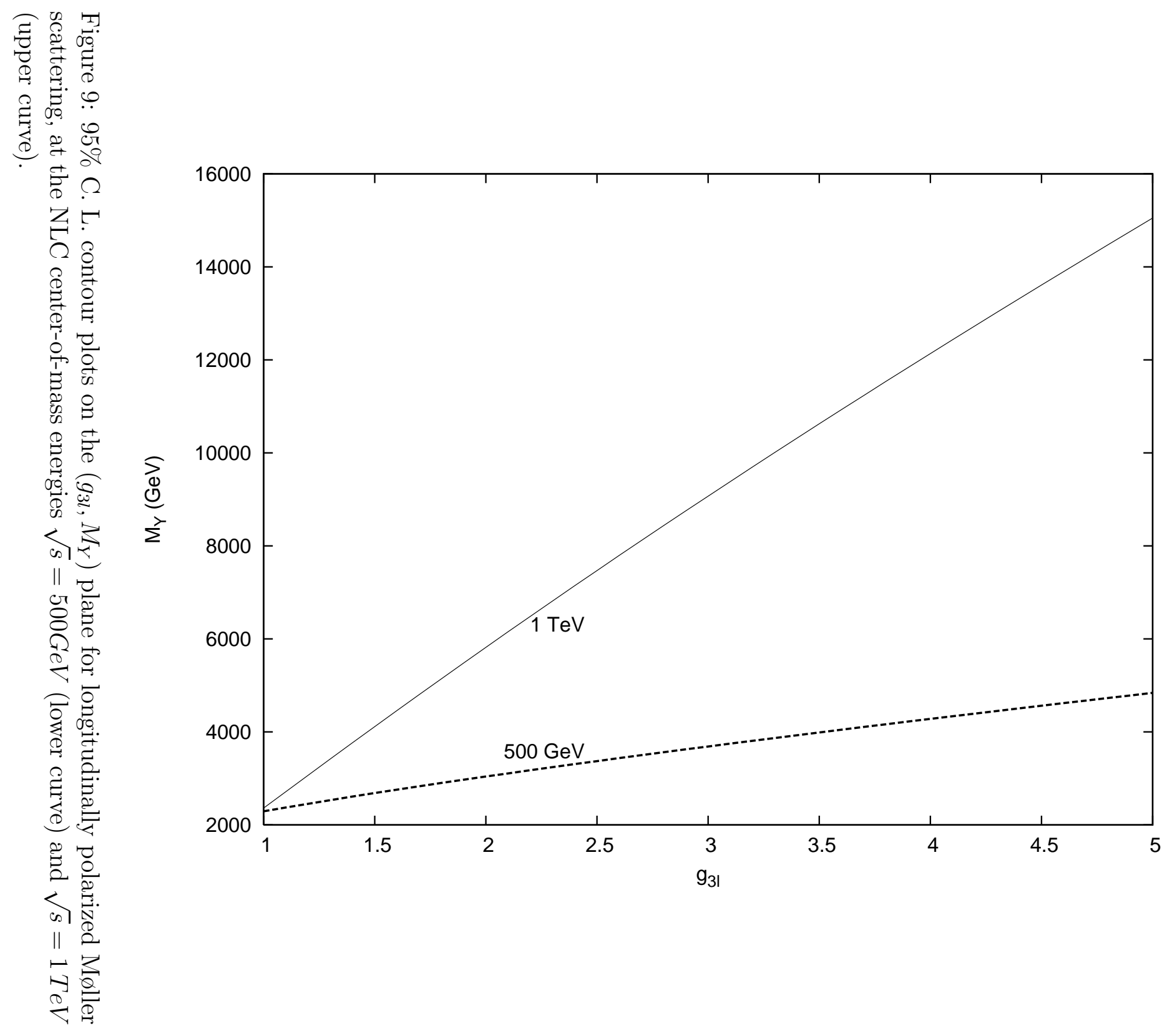




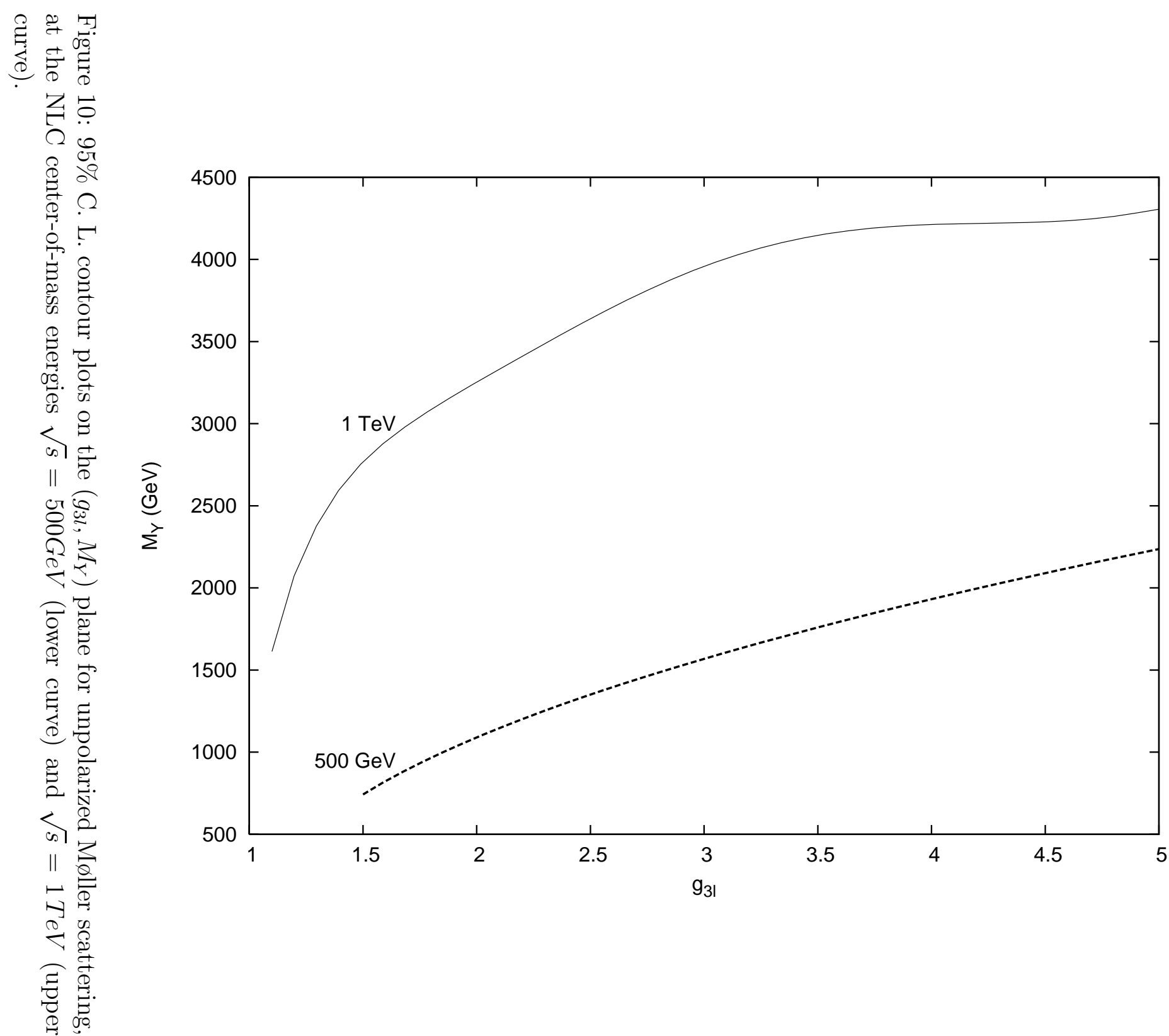

